\title{
SPECTROSCOPIC OBSERVATIONS OF THE LONG-PERIOD WOLF-RAYET BINARIES HD 193793 AND HD 192641
}

\author{
K. ANNUK \\ Tartu Astrophysical Observatory, EE 2444 Töravere, Estonia
}

\begin{abstract}
New medium-resolution spectroscopic observations of two long-period WR binaries have been obtained using the $1.5 \mathrm{~m}$ telescope at the Tartu Astrophysical Observatory. The binarity of these systems is confirmed and orbital parameters are determined.
\end{abstract}

Key words: Wolf-Rayet stars - Binaries - Radial velocities

\section{Introduction}

The prototype of the stars showing strong IR variations and dust formation is HD 193793 (WC7+04-06). Its binarity was doubted for a long time. The first attempt to determine an orbit was made by Lamontagne et al. (1984) but Conti et al. (1984) found no orbital motion. Some years later Williams et al. (1987b) detected from IR photometry the period of $2885 \mathrm{~d}$. This period was confirmed in further investigations by Moffat et al. (1987), Williams et al. (1990) and Annuk (1991). Another outstanding star of this class is HD 192641 (WC7+09). Previous radial velocity measurements by Massey et al. (1981) and Moffat et al. (1986) have not given any periods. From IR photometry Williams et al. (1987a) suggested that the possible period is about $4400 \mathrm{~d}$. This period was supported by our radial velocity measurements (Annuk 1991). Recently, Underhill (1992) put its binarity under suspicion claiming that radial velocities were constant.

\section{Obervations}

The spectroscopic observations were carried out at the Tartu Astrophysical Observatory using a $1.5 \mathrm{~m}$ telescope with a Cassegrain spectrograph ASP32 (at a reciprocal dispersion of $37 \AA / \mathrm{mm}$ or $18 \AA / \mathrm{mm}$ ). Radial velocities of the strongest emission line, $\mathrm{C}$ IV $\lambda 4650$ were computed by the method of bisector. Radial velocities of other emission lines and absorption lines were determined by fitting the Gaussian profiles or the parabola upon the normalized intensity profiles.

\section{HD 193793}

New additional spectra were obtained around the last periastron passage in the years 1991-93. Radial velocities of absorption were calculated as a mean of several strong absorption lines. Typical $1 \sigma$ limits of averaging were $8-10 \mathrm{~km} / \mathrm{s}$. Radial velocities of the emission line C IV $\lambda 4650$ were calculated as a mean of the levels $I / I_{c}=0.55-0.80(1 \sigma=10-15 \mathrm{~km} / \mathrm{s})$. Around the peri- 


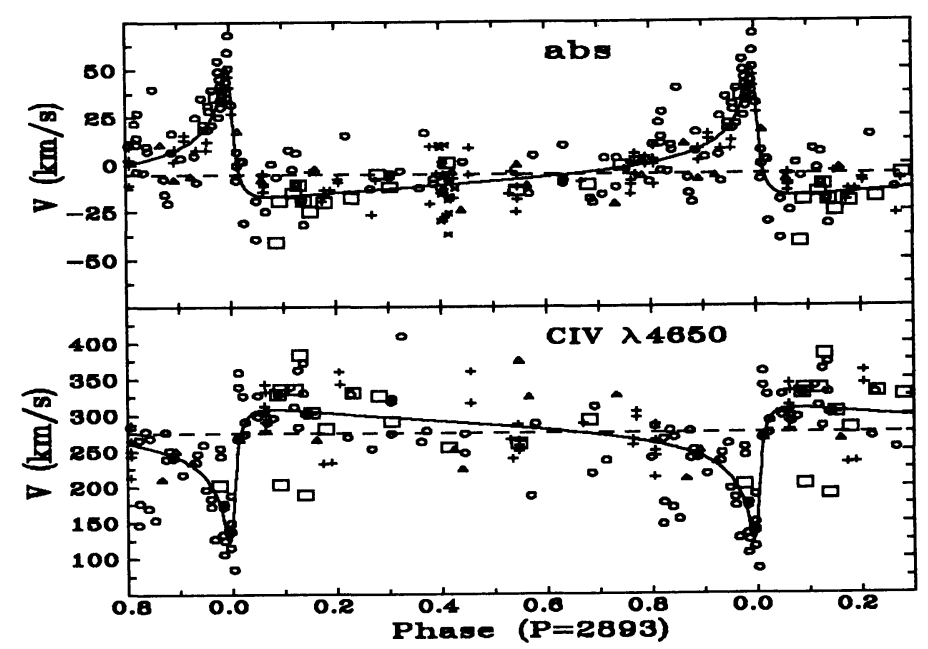

Fig. 1. Velocity curves of HD 193793. - - this paper; + - Conti et al. (1984); $\Pi-$ Lamontagneet al. (1984); $\Delta$ - Moffat et al. (1987); $\times$ - Galkina (1970).

astron passage we can see clear antiphase variations of the absorption line velocities $\left(v_{a b s}\right)$ and emission line velocities $\left(v_{e m}\right)$. Immediately after the periastron passage the $v_{a b s}$ very quickly decrease but $v_{e m}$ increase. During a month $\Delta v \approx-50 \mathrm{~km} / \mathrm{s}$ for absorption and $\Delta v \approx 140 \mathrm{~km} / \mathrm{s}$ for emission. Such variations very strongly support the hypothesis of a very eccentric ( $e$ $=0.85$ ) binary system. By using our new and all available observations we derived new orbital elements (see Table 1). The corresponding radial velocity curves are plotted in Figure 1. Our new elements do not differ from those found earlier by Williams et al. (1990) and by us (Annuk 1991). We found that the last periastron passage took place on February $17( \pm 4), 1993$. Using the infrared data (e.g. Williams et al. 1990) we can conclude that the IR maximum (at $3.8 \mu \mathrm{m}$ ) appears about 50 days (in phase $\sim 0.017$ ) after the periastron passage. The minimum distance between the $\mathrm{WR}$ and the $\mathrm{O}$ component is $d \sin i \approx 568 \mathrm{R}_{\odot}$ and the mass ratio $q=0.37 \pm 0.04$.

\section{HD 192641}

Radial velocities of the strongest emission line were calculated as a mean of the levels $\mathrm{I} / \mathrm{I}_{c}=0.45-0.70(1 \sigma=7-11 \mathrm{~km} / \mathrm{s})$. We were able to measure radial velocities of some other emission lines (e.g. C IV $\lambda 4441, \mathrm{C}$ IV $\lambda 4786$ and $\mathrm{C}$ III $\lambda 4069)$ but the accuracy was somewhat worse. Although the absorption lines are weak we have measured the radial velocities from better spectrograms. $1 \sigma$ limits for the absorptions were $20-30 \mathrm{~km} / \mathrm{s}$. Our new radial velocities of the line C IV $\lambda 4650$ do not fit with our previous orbital elements (Annuk 1991). If we assume that the period is about $4400 \mathrm{~d}$ as it was indicated from 
TABLE I

Orbital elements of HD 193793.

\begin{tabular}{lll}
\hline Element & abs & CIV $\lambda 4650$ \\
\hline$P($ day $)$ & 2893 & 2893 \\
$e$ & $0.85 \pm 0.01$ & 0.85 \\
$\gamma(\mathrm{km} / \mathrm{s})$ & $-5.1 \pm 0.5$ & $275.5 \pm 2.3$ \\
$K(\mathrm{~km} / \mathrm{s})$ & $33.8 \pm 1.5$ & $91.3 \pm 4.8$ \\
$\omega\left(^{\circ}\right)$ & $40.8 \pm 3.1$ & 220.8 \\
$\mathrm{~T}_{0}(2440000)$ & $9036 \pm 4$ & $9034 \pm 5$ \\
$a \sin i\left(\mathrm{R}_{\odot}\right)$ & $1022 \pm 46$ & $2750 \pm 144$ \\
$m \sin ^{3} i\left(\mathrm{M}_{\odot}\right)$ & $62.7 \pm 9.6$ & $23.2 \pm 3.4$ \\
$\sigma(\mathrm{km} / \mathrm{s})$ & 8.6 & 32.5 \\
\hline
\end{tabular}

TABLE II

Orbital elements of HD 192641.

\begin{tabular}{lll}
\hline Element & CIV $_{\text {IV }} 4650$ & abs \\
\hline$P($ day $)$ & 5680 & \\
$e$ & $0.07 \pm 0.03$ & 0.07 \\
$\gamma(\mathrm{km} / \mathrm{s})$ & $80.7 \pm 0.7$ & $0.2 \pm 2.3$ \\
$K(\mathrm{~km} / \mathrm{s})$ & $30.5 \pm 1.1$ & $21.6 \pm 3.7$ \\
$\omega\left(^{\circ}\right)$ & $380 \pm 24$ & 200 \\
$\mathrm{~T}_{0}(2440000)$ & $6017 \pm 380$ & 6017 \\
$f(m)\left(\mathrm{M}_{\odot}\right)$ & $16.6 \pm 1.7$ & \\
$\sigma(\mathrm{km} / \mathrm{s})$ & 6.4 & 18.7 \\
\hline
\end{tabular}

an earlier infrared curve then the orbit must be more eccentric $(e \geq 0.55)$. Thus in this case there is a slight disagreement with the velocities measured by Moffat et al. (1986) in 1981 and with our recent measurements. Another way is to assume that the period is longer than $4400 \mathrm{~d}$. In this case there is a disagreement in the infrared curve, although new IR measurements indicate a longer period (Williams 1994, these proceedings). From all available data we found a new possible period of $5680 \mathrm{~d}$. Our new derived orbital elements are given in Table 2 and the corresponding curves are plotted in Figure 2. In this Figure we have also plotted the radial velocities of the measurements of Underhill (1992). It must be said that her velocities of the line C IV $\lambda 4650$ coincide very nicely with our velocities. We need to add only some correction of about $70 \mathrm{~km} / \mathrm{s}$ to Underhill's velocities for bringing her data into good agreement with our values. This correction is mainly caused by the fact that she calculated the velocities with respect to $\lambda 4651.56$ but we with respect to $\lambda 4650.16$. As we can see from Figure 2, the absorption line velocities are very scattered and therefore the solution for absorption is quite uncertain. 


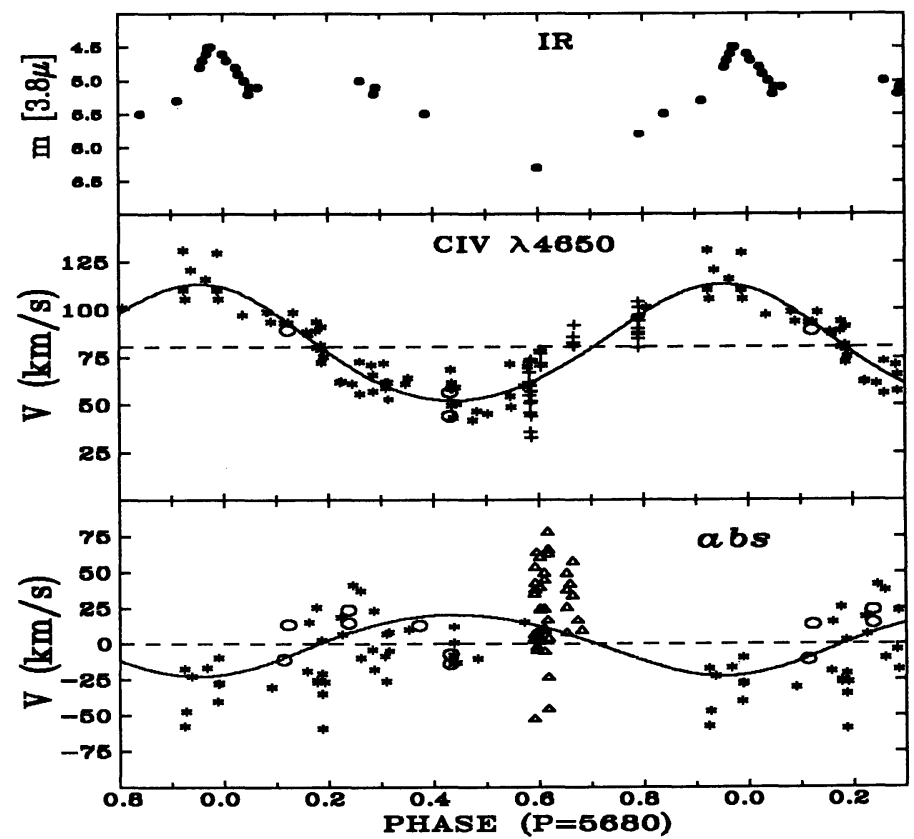

Fig. 2. Velocity curves of HD 192641. * - this paper; + - Moffat et al. (1986); $\Delta$ Massey et al. (1981). IR photometry based on the paper of Williams et al. (1987a).

More observations are needed for this star.

\section{References}

Annuk, K. 1991, in K.A. van der Hucht \& B. Hidayat (eds.) Wolf-Rayet Stars and Interrelations with Other Massive Stars in Galxies, Proc. IAU Symp. No. 143, (Dordrecht: Kluwer), p. 245

Conti, P.S., Roussel-Dupre, D., Massey, P., Rensing, M. 1984, ApJ 282, 693

Galkina, T.S. 1970, Izvestiya Krymskoi Astrof. Obs. 41-42, 283

Lamontagne, R., Moffat, A.F.J., Seggewiss, W. 1984, $A p J$ 277, 258

Massey, P., Conti, P.S., Niemela, V.S. 1981, ApJ 246, 145

Moffat, A.F.J., Lamontagne, R., Shara, M.M., McAlister, H.A. 1986, AJ 91, 1392

Moffat, A.F.J., Lamontagne, R., Williams, P.M., Horn, J., Seggewiss, W. 1987, ApJ 312 , 807

Underhill, A. 1992, $A p J$ 398, 636

Williams, P.M., van der Hucht, K.A., Thé, P.S. 1987a, QJRAS 28, 248

Williams, P.M., van der Hucht, K.A., van der Woerd, H., Wamsteker, W.M., Geballe, T.R., Garmany, C.D., Pollock, A.M.T. 1987b, in: H. Lamers and C. de Loore (eds.), Instabilities in Luminous Early-Type Stars (Dordrecht: Reidel), p. 221

Williams, P.M., van der Hucht, K.A., Pollock, A.M.T., Florkowski, D.R., van der Woerd, H., Wamsteker, W.M. 1990, MNRAS 243, 662

\section{DISCUSSION:}

Moffat: Do you see any phase-dependent time profile variations, especially around periastron passage, when wind interaction effects are expected to be important?

Annuk: No, we didn't find such variations. 